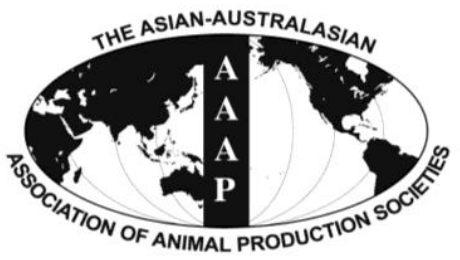

Asian Australas. J. Anim. Sci.

Vol. 26, No. 10 : 1416-1423 October 2013

http://dx.doi.org/10.5713/ajas.2013.13134

www.ajas.info

pISSN 1011-2367 elSSN 1976-5517

\title{
Production of Citrate by Anaerobic Fungi in the Presence of Co-culture Methanogens as Revealed by ${ }^{1} \mathrm{H}$ NMR Spectrometry
}

\author{
Yan Fen Cheng, Wei Jin, Sheng Yong Mao, and Wei-Yun Zhu* \\ Laboratory of Gastrointestinal Microbiology, Nanjing Agricultural University, Nanjing, Jiangsu, 210095, China
}

\begin{abstract}
The metabolomic profile of the anaerobic fungus Piromyces sp. F1, isolated from the rumen of goats, and how this is affected by the presence of naturally associated methanogens, was analyzed by nuclear magnetic resonance spectroscopy. The major metabolites in the fungal monoculture were formate, lactate, ethanol, acetate, succinate, sugars/amino acids and $\alpha$-ketoglutarate, whereas the co-cultures of anaerobic fungi and associated methanogens produced citrate. This is the first report of citrate as a major metabolite of anaerobic fungi. Univariate analysis showed that the mean values of formate, lactate, ethanol, citrate, succinate and acetate in co-cultures were significantly higher than those in the fungal monoculture, while the mean values of glucose and $\alpha$-ketoglutarate were significantly reduced in co-cultures. Unsupervised principal components analysis revealed separation of metabolite profiles of the fungal mono-culture and co-cultures. In conclusion, the novel finding of citrate as one of the major metabolites of anaerobic fungi associated with methanogens may suggest a new yet to be identified pathway exists in co-culture. Anaerobic fungal metabolism was shifted by associated methanogens, indicating that anaerobic fungi are important providers of substrates for methanogens in the rumen and thus play a key role in ruminal methanogenesis. (Key Words: Piromyces sp. F1, Methanobrevibacter thaueri, Nuclear Magnetic Resonance, Citrate)
\end{abstract}

\section{INTRODUCTION}

Obligate anaerobic fungi have only been reported in gastrointestinal and fecal communities since their first discovery in 1975 (Orpin, 1975), and have been found to exist exclusively in herbivores, including ruminants and hindgut-fermenting animals (Theodorou et al., 1995). These fungi play an important role in the primary digestion of plant fiber via a broad range of highly active fiberdegrading enzymes and their extensive rhizoid system (Theodorou et al., 1995). The metabolites of anaerobic fungi have so far been reported as formate, acetate, lactate, ethanol, succinate, $\mathrm{CO}_{2}$ and $\mathrm{H}_{2}$ (Bauchop and Mountfort, 1981), but no comprehensive metabolomic profiles of anaerobic fungi have been reported to date.

Methanogenic archaea comprise one of the important functional groups in the rumen and can utilize hydrogen, formate and acetate, metabolites of anaerobic fungi, to produce methane (Hungate, 1982). Previous studies showed that co-cultures of different anaerobic fungi and

\footnotetext{
* Corresponding Author: Wei-Yun Zhu. Tel: +86 25 84395523, Fax: +86 25 84395314, E-mail: zhuweiyun@njau.edu.cn Submitted Mar. 4, 2013; Accepted Apr. 30, 2013; Revised May 26, 2013
}

methanogens result in increased acetate formation and decreased ethanol, lactate, and formate production (Bauchop and Mountfort, 1981; Marvin-Sikkema et al., 1990; Nakashimada et al., 2000), but these co-cultures were simple mixtures of axenic pure cultures of an anaerobic fungus and a methanogen. In order to provide a more natural model system, more closely mimicking the interactions of anaerobic fungi and methanogens in the rumen ecosystem, Cheng et al. $(2006 ; 2009)$ obtained a mixed natural co-culture of anaerobic fungi and methanogens from the goat rumen and found that the formate and lactate were reduced in the mixed co-culture and acetate was increased. Subsequently, Jin et al. (2011) obtained a natural simple co-culture of anaerobic fungus and its indigenously associated methanogen from the previous mixed co-culture in order to investigate the metabolic interaction of anaerobic fungi and methanogens in the rumen and their abilities to degrade lignocellulosic materials and to produce methane.

Nuclear magnetic resonance (NMR) spectroscopy is a powerful analytical technique for metabolomic studies, providing unique and rich information content with high sensitivity and reproducibility (Beckonert et al., 2007). It has been widely used as a fingerprinting tool for metabolic 
studies of microorganisms including bacteria and yeast (Grivet and Delort, 2009). Metabolites generally associated with rumen anaerobic fungi include formate, acetate, lactate, succinate, ethanol, $\mathrm{CO}_{2}$ and $\mathrm{H}_{2}$ (Theodorou et al., 1996). Little information is available on other metabolites of rumen anaerobic fungi. In this study, we hypothesized the existence of other metabolites produced by rumen anaerobic fungi and that the presence of co-cultured methanogens could influence the metabolic profiles of rumen anaerobic fungi. To test this hypothesis, NMR was used to compare the metabolic profiles of three cultures, an anaerobic fungal monoculture Piromyces sp. F1, a simple naturally occurring co-culture of Piromyces sp. F1 and its indigenously associated methanogen Methanobrevibacter thaueri, and a mixed co-culture of anaerobic fungi and methanogens derived from rumen digesta.

\section{MATERIALS AND METHODS}

\section{Microbial cultures}

Three cultures were used in this study: i) a mixed coculture comprising a diverse range of anaerobic fungi and methanogens derived from a goat rumen (Cheng et al., 2006); ii) a natural simple co-culture of an anaerobic fungus, Piromyces sp. F1, and an associated methanogen, Methanobrevibacter thaueri, obtained from this mixed coculture using the Hungate roll-tube method (Jin et al., 2011); iii) a pure culture of Piromyces sp. F1 derived from the simple co-culture by adding chloramphenicol to inhibit growth of the methanogen.

\section{Samples collection}

The cultures were grown anaerobically at $39^{\circ} \mathrm{C}$ in 100 $\mathrm{mL}$ defined medium M2 (Teunissen et al., 1991) supplemented with $1 \%$ cellobiose. Experimental cultures were inoculated by transferring $10 \mathrm{~mL}$ of a $3 \mathrm{~d}$-old culture to $90 \mathrm{~mL}$ medium $\mathrm{M} 2$. After incubation at $39^{\circ} \mathrm{C}$ for $3 \mathrm{~d}$, the liquid fractions of the cultures were collected, centrifuged at $15,500 \mathrm{~g}$ and the supernatant were stored at $-20^{\circ} \mathrm{C}$ for NMR analysis.

\section{Chemicals and sample preparation for NMR analysis}

$\mathrm{D}_{2} \mathrm{O}(99.9 \%)$ and TSP (3-(trimethylsilyl) propionic2,2,3,3- $d_{4}$-acid, sodium salt) were purchased from Cambridge Isotope Laboratories (Miami, FL, USA) and Merck (Darmstadt, Germany), respectively. Culture supernatant $(400 \mu \mathrm{L})$ was mixed with $100 \mu \mathrm{L}$ TSP/D $2 \mathrm{O}(1$ $\mathrm{mg} / \mathrm{mL}$ ), centrifuged at $15,500 \mathrm{~g}$ for $5 \mathrm{~min}$ and the supernatant was transferred into NMR tubes.

\section{NMR analysis}

${ }^{1} \mathrm{H}$ NMR spectra were obtained on an INOVA $600 \mathrm{MHz}$ spectrometer (Varian, USA). The residual water signal was suppressed by the pre-saturation method (Lu et al., 2010). 1D ${ }^{1} \mathrm{H}$ NMR spectra were acquired with the following parameters: spectral width $8000 \mathrm{~Hz}$, mix time $0.1 \mathrm{~s}$, relaxation delay $2 \mathrm{~s}, 32 \mathrm{~K}$ complex data points, 64 transients. The data were zero-filled by a factor of 4 and the free induction decays (FIDs) were multiplied by an exponential weighting function equivalent to a line boarding of $0.5 \mathrm{~Hz}$ prior to Fourier transformation (FT). The peak of external TSP was designated as $0.00 \mathrm{ppm}$ as baseline, and the chemical shifts of other chemicals were referenced to the peak of external TSP.

\section{NMR data processing}

The ${ }^{1} \mathrm{H}$ NMR spectra were manually phased and the baseline was corrected and referenced to TSP $(\delta=0)$. The spectral region $(\delta=0.80-8.80)$ was segmented into 200 regions at $0.04 \mathrm{ppm}$ per interval. The region from 4.60 to 5.00 was excluded from the analysis because of the residual signal of water in aqueous extracts. The integrated data were normalized to the total integrals of each spectrum for pattern recognition analysis (Wang et al., 2004) and the data were transferred into an excel file.

\section{Identification of metabolites}

Identification of metabolites was based on their chemical shifts and multiplicities with the assistance of previous publications and publicly available metabolite libraries from the NMR metabolomics database of Linkoping, Sweden (MDL) (http://www.liu.se/hu/mdl/ main/).

\section{Data analysis}

${ }^{1} \mathrm{H}$ NMR spectra data were analyzed using the SIMCA$\mathrm{P}$ software package (Umetris $\mathrm{AB}$, Umea, Sweden) for multivariate statistical analysis. All the variables were mean-centered and Pareto-scaled prior to principal components analysis. The statistical significance of differences between mean values of metabolites was analyzed by SPSS software (SPSS Inc., Chicago, USA).

\section{RESULTS AND DISCUSSION}

\section{NMR spectra and assignment of compounds}

After incubation at $39^{\circ} \mathrm{C}$ for $3 \mathrm{~d}$, the three cultures grew well. Methane was detected in the gas phase of the two cocultures, while no methane was detected from the monoculture.

The NMR spectra of water-soluble metabolites from suspensions of three cultures are shown in Figure 1. The major metabolites in the anaerobic fungal monoculture were formate $(\delta=8.46)$, acetate $(\delta=1.94)$, lactate $(\delta=1.34)$, 

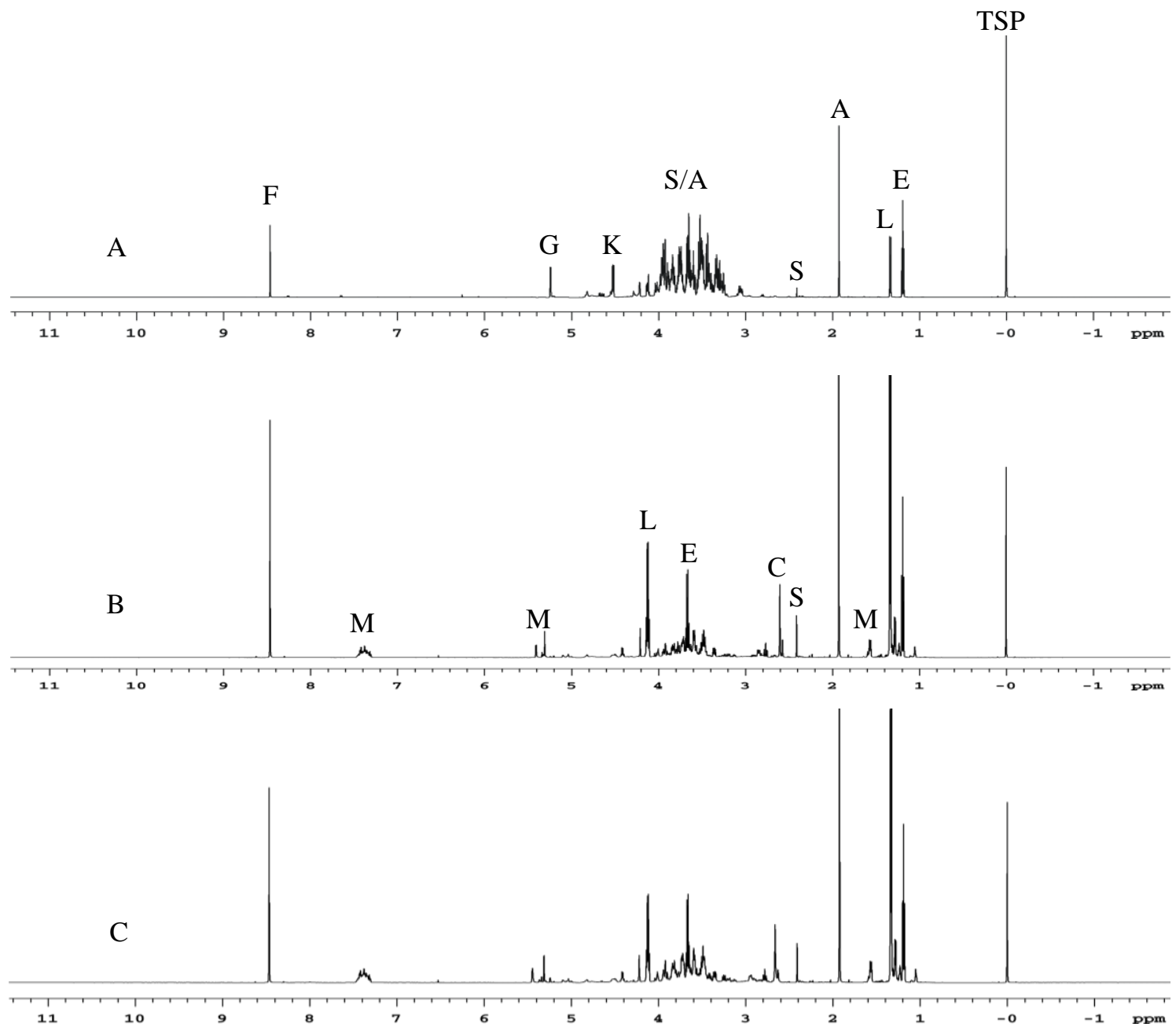

Figure 1. Nuclear magnetic resonance (NMR) spectra of the water-soluble metabolites from suspensions of anaerobic fungal monoculture (Piromyces sp. F1; A), a simple co-culture of anaerobic fungus and associated methanogen co-culture (Piromyces sp. $\mathrm{F} 1+$ Methanobrevibacter thaueri; B) and a mixed co-culture of anaerobic fungi and methanogens derived from rumen digesta, from which the simple co-culture was obtained (C). ${ }^{1} \mathrm{H}$ NMR spectra were obtained on an INOVA $600 \mathrm{MHz}$ spectrometer (Varian, USA). The ${ }^{1} \mathrm{H}$ NMR spectra were manually phased and the baseline was corrected and referenced to TSP $(\delta=0)$. The spectral region $(\delta=0.80-8.80)$ was segmented into 200 regions at 0.04 ppm per intervals. The region from 4.60 to 5.00 was excluded from the analysis because of the residual signal of water in aqueous extracts. $\mathrm{A}=$ Acetate; $\mathrm{C}=$ Citrate; $\mathrm{E}=$ Ethanol; $\mathrm{F}=$ Formate; $\mathrm{G}=\mathrm{Glucose} ; \mathrm{L}=\mathrm{Lactate} ; \mathrm{M}=$ Unidentified medium components; $\mathrm{S}=$ Succinate; $\mathrm{K}=\alpha$-ketoglutarate; $\mathrm{S} / \mathrm{A}=$ Sugars/amino acids; TSP $=$ Internal reference, 3 (trimethylsilyl) propionic-2,2,3,3-d4-acid.

ethanol $(\delta=1.18)$, sugars/amino acids $(\delta=3.2-4.0)$ and $\alpha$-ketoglutarate $(\delta=4.54$ ) (Kwon et al., 2009). Succinate $(\delta$ $=2.42$ ), a common metabolite of anaerobic fungi, was detected in low amounts. Glucose $(\delta=5.22)$, derived by anaerobic fungal exoenzymes from cellobiose, was detected in large amounts in the culture.

In the present study, $\alpha$-ketoglutarate was detected for the first time, supporting the assumed metabolic pathway of anaerobic fungi proposed by Kwon et al. (2009). Kwon et al. (2009) constructed an EST library of rumen fungus Neocallimastix frontalis. According to the detected genes for glucose metabolism, they elucidated the fungal carbohydrate metabolism pathway and postulated that the metabolites for Neocallimastix frontalis were acetate, ethanol, formate, lactate, succinate and $\alpha$-ketoglutarate, which was oxidized from oxaloacetate by aconitase and isocitrate dehydrogenase. In other previous studies, the metabolites of anaerobic fungi were reported as formate, acetate, lactate, ethanol and succinate. Boxma et al. (2004) calculated the relative fluxes of glucose through known metabolic pathways and demonstrated that the major fermentation end-products of anaerobic fungi were lactate, formate, ethanol, acetate and succinate. In the present study lactate, formate, ethanol, acetate were detected as major fermentation end-products, while succinate was detected in low amounts, accounting for $0.16 \%$ of the total metabolites. 
Glucose was detected in the supernatant of the fungal monoculture, suggesting that the substrate was degraded incompletely in this culture. Large amounts of sugars/amino acids were also detected in the supernatant but were difficult to separate and identify as reported by Chikayama et al. (2008). Chikayama et al. (2008) compiled a heteronuclear single quantum coherence-based metabolite chemical shift database that contains only NMR spectra of standard compounds measured under standardized conditions. This database is a compilation of $1,018{ }^{1} \mathrm{H}$ and ${ }^{13} \mathrm{C}$ chemical shifts corresponding to 142 metabolites, showing that many sugars, nucleic acids and organic acids were distributed within a narrow range of chemical shifts between 3 and 4 ppm for ${ }^{1} \mathrm{H}$ NMR.

It is interesting that apart from the major metabolites formate, acetate, lactate, ethanol and succinate as reported previously, citrate $(\delta=2.66)$ was for the first time demonstrated as one of the major metabolites in co-cultures. In these co-cultures, methanogens can only use simple compounds such as $\mathrm{H}_{2} / \mathrm{CO}_{2}$, formate, acetate etc. to produce methane, and Methanobrevibacter sp. strains extensively use $\mathrm{H}_{2} / \mathrm{CO}_{2}$ or/and formate as substrate to produce methane (Balch et al., 1979). But the mechanism of citrate production by anaerobic fungi is unknown and needs to be further investigated. Meanwhile, two peaks at $4.14 \mathrm{ppm}$ and $3.66 \mathrm{ppm}$ chemical shift, representing lactate and ethanol respectively, were observed in the co-cultures. Other peaks at $7.38 \mathrm{ppm}, 4.42 \mathrm{ppm}, 2.78 \mathrm{ppm}, 1.58 \mathrm{ppm}$ and $1.26 \mathrm{ppm}$, were not observed in the fungal monoculture, and were found, by NMR analysis, to come from the growth medium (data not shown).

The mean values of the metabolites from three cocultures are shown in Table 1; suggesting that the concentrations of formate, acetate, lactate, citrate, succinate and ethanol were significantly higher in co-cultures than the anaerobic monoculture. But, there was no statistical difference between the concentrations of these metabolites in the two co-cultures, implying similar fungal metabolism in the two co-cultures. In agreement with the suggestion by Kwon et al. (2009), $\alpha$-ketoglutarate was one of the major metabolites in the fungal mono-culture. However, the concentration of $\alpha$-ketoglutarate was similarly low in the two co-cultures, significantly lower than in the anaerobic fungal monoculture. Meanwhile, sugars/amino acids within 3.0 to $4.0 \mathrm{ppm}$, except ethanol ( $\delta=3.66 \mathrm{ppm})$, were found at much lower concentrations in the co-cultures than in the fungal monoculture. Glucose split by anaerobic fungal exoenzymes from cellobiose was also detected at significantly lower concentration in co-cultures than the fungal monoculture. This may imply that the substrate for anaerobic fungi was utilized completely in the co-cultures. Consequently, the production of metabolites such as formate, acetate, ethanol, lactate and succinate was increased in these co-cultures.

Bauchop and Mountfort (1981) reported that the amount of acetate in culture supernatant was much higher when the anaerobic fungus Neocallimastix strain PN1 was cocultured with Methanobrevibacter sp. strain RA1 than in the fungal monoculture. The authors subsequently reported that when Methanosarcina barkeri was cultured with Neocallimastix strain PN1 and Methanobrevibacter sp. strain RA1 co-culture, acetate could not be detected in the tri-culture (Mountfort et al., 1982), suggesting that the amount of acetate produced in the co-culture of anaerobic fungi and methanogens was dependent on the co-cultured methanogen species. However, these authors reported that lactate, ethanol and formate were produced at much lower levels in the co-culture than in fungal mono-culture (Bauchop and Mountfort, 1981; Mountfort et al., 1982).

Table 1. Mean values of detected metabolites in an anaerobic fungal mono-culture and two fungal/methanogen co-cultures

\begin{tabular}{|c|c|c|c|c|}
\hline \multirow{2}{*}{$\begin{array}{l}\text { Chemical shifts }(\delta)^{1} \\
(\mathrm{ppm})\end{array}$} & \multirow{2}{*}{ Compounds $^{2}$} & \multicolumn{3}{|c|}{ Cultures $^{3}$} \\
\hline & & A & B & $\mathrm{C}$ \\
\hline 1.18 & Ethanol & $291.5 \pm 20.3^{\mathrm{a}, 4}$ & $489.2 \pm 24.4^{\mathrm{b}}$ & $429.9 \pm 51.6^{\mathrm{b}}$ \\
\hline 1.34 & Lactate & $186.2 \pm 8.0^{\mathrm{a}}$ & $1984.9 \pm 124.5^{\mathrm{b}}$ & $1434.9 \pm 436.7^{\mathrm{b}}$ \\
\hline 1.94 & Acetate & $204.2 \pm 10.2^{\mathrm{a}}$ & $710.4 \pm 2.6^{\mathrm{b}}$ & $653.5 \pm 94.8^{\mathrm{b}}$ \\
\hline 2.42 & Succinate & $15.8 \pm 1.1^{\mathrm{a}}$ & $75.7 \pm 0.7^{\mathrm{b}}$ & $65.7 \pm 11.4^{\mathrm{b}}$ \\
\hline 2.66 & Citrate & $19.2 \pm 1.9^{\mathrm{a}}$ & $283.0 \pm 18.2^{\mathrm{b}}$ & $248.0 \pm 10.1^{\mathrm{b}}$ \\
\hline 4.54 & $\alpha$-Ketoglutarate & $145.4 \pm 5.4^{\mathrm{a}}$ & $27.1 \pm 0.3^{b}$ & $39.2 \pm 10.4^{b}$ \\
\hline 5.22 & Glucose & $112.5 \pm 5.4^{\mathrm{a}}$ & $9.8 \pm 0.5^{\mathrm{b}}$ & $23.3 \pm 10.2^{\mathrm{b}}$ \\
\hline 8.46 & Formate & $115.6 \pm 2.1^{\mathrm{a}}$ & $429.3 \pm 16.1^{\mathrm{b}}$ & $315.7 \pm 62.7^{\mathrm{b}}$ \\
\hline
\end{tabular}

${ }^{1}$ The baseline was corrected and referenced to TSP (3-(trimethylsilyl) propionic-2,2,3,3- $d_{4}$-acid, sodium salt; $\delta=0$ ).

${ }^{2}$ Identification of metabolites was based on their chemical shifts and multiplicities with the assistance of previous publications and publicly available metabolite libraries from the NMR metabolomics database of Linkoping, Sweden (MDL) (http://www.liu.se/hu/mdl/main/).

${ }^{3} \mathrm{~A}=$ Anaerobic fungal monoculture; $\mathrm{B}=\mathrm{Co}$-culture of anaerobic fungus and methanogen; $\mathrm{C}=$ Mixed co-culture of anaerobic fungi and methanogens derived from the rumen. Mean values are presented as mean \pm SEM.

${ }^{4}$ The statistical significance of differences between mean values of metabolites was analyzed by SPSS software (SPSS Inc., Chicago, USA). In each row, values followed by different superscripts are statistically significant $(\mathrm{p}<0.05)$. 

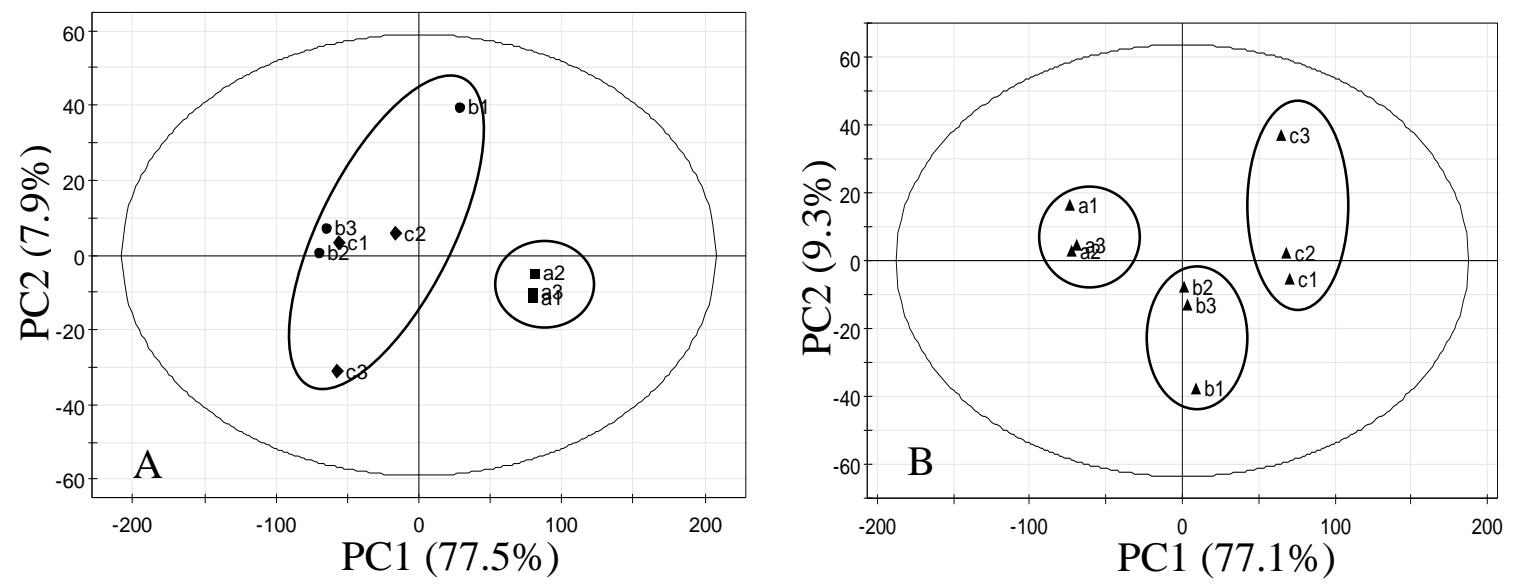

Figure 2. PLS-DA and O-PLS analysis derived score plots of the first two principal components (PC1 and PC2) of suspensions of an anaerobic fungal mono-culture (a1-3), a simple co-culture of an anaerobic fungus and methanogen (b1-3) and a mixed co-culture of anaerobic fungi and methanogens derived from goat rumen digesta (c1-3). The ${ }^{1} \mathrm{H}$ NMR spectra data were analyzed using the SIMCA-P software package (Umetris AB, Umea, Sweden). All the variables were mean-centered and Pareto-scaled prior to principal components analysis and the ellipse represents the $95 \%$ confidence region.

Similarly, Joblin et al. (1990) reported that levels of lactate and formate were much lower in a co-culture of $N$. frontalis and Methanobrevibacter smithii than fungal mono-culture. Cheng et al. (2009) also reported a much lower lactate and formate concentrations in mixed co-culture of anaerobic fungi and methanogens than fungal culture. These reports contrast with data reported here, which demonstrated that formate, acetate, ethanol and lactate all increased in cocultures. These differences may be due to the different substrates used in different studies. Jin et al. (2011) investigated the same co-cultures as that used in this study and reported that there were no significant differences on the levels of lactate and ethanol between co-culture and fungal culture, when growing on corncob. Our other study on the same co-culture showed that the acetate, ethanol and lactate all significantly increased in co-cultures, when growing on cassave (Liu, 2009). Thus, this metabolic differences on different substrates might be dependent on anaerobic fungal and methanogen species.

It should also be noted that in previous studies, culture metabolites were assayed by chemical methods that analyzed individual compounds (Mountfort et al., 1982; Cheng et al., 2009), while the NMR analysis used here is more sensitive and reliable. NMR has been used to investigate microbial physiology and metabolism for over 30 yrs (Grivet, 2001). Carrieri et al. (2009) used this approach to demonstrate that the water-soluble metabolites of two cyanobacterial species Arthrospora maxima and Synechococcus 7002 were formate, lactate, ethanol, acetate and succinate. Pham et al. (2005) identified the secondary metabolites from Streptomyces violaceoruber and results showed that more than $50 \%$ of the identified metabolites were new compounds.

\section{Principle components analysis}

The data were analyzed with PLS-DA and O-PLS (Figure 2A and 2B), but only O-PLS could discriminate the three cultures, and the first two principal components cumulatively accounted for $86.3 \%$ of the total variation. O-PLS was then used to investigate the major compounds that contributed to discrimination, and an O-PLS derived variable importance plot is shown in Figure 3. The most

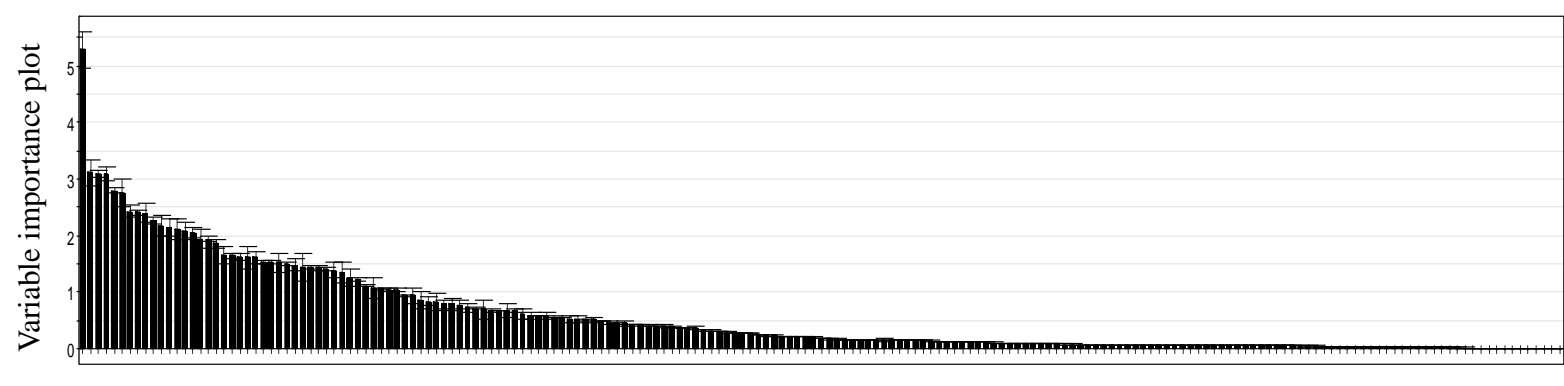

Variable ID (chemical shift)

Figure 3. O-PLS derived variable importance plot of the suspensions of an anaerobic mono-culture and two fungal/methanogen cocultures. The variable ID corresponds to a chemical shift (ppm). 
important compound contributing to the discrimination was lactate, corresponding to a chemical shift $1.34 \mathrm{ppm}$, followed by acetate, sugars/AA, ethanol and formate (Table 2).

\section{Metabolic pathway of anaerobic fungi with/withou methanogens}

According to previous reports, lactate, formate, ethanol and succinate were formed in the cytosol of anaerobic fungi, and acetate, formate and $\mathrm{H}_{2} / \mathrm{CO}_{2}$ were formed in the hydrogenosome (O'Fallon et al., 1991; Akhmanova et al., 1999; Boxma et al., 2004). In a recent report by Kwon et al. (2009), $\alpha$-ketoglutarate was proposed to be produced in the cytosol. In the present study, citrate was reported as metabolite of anaerobic fungi in co-culture with methanogens. Based on these reports, a modified metabolic pathway of glucose by anaerobic fungal monoculture is
Table 2. The five major compounds contributing to O-PLS clustering of an anaerobic fungal mono-culture and two fungal/methanogen co-cultures

\begin{tabular}{lccl}
\hline No. & $\begin{array}{c}\text { Chemical shift }(\delta) \\
(\mathrm{ppm})\end{array}$ & VIP $^{1}$ value & $\begin{array}{c}\text { Corresponding } \\
\text { metabolites }\end{array}$ \\
\hline 1 & 1.34 & 5.28 & Lactate \\
2 & 1.94 & 3.11 & Acetate \\
3 & 3.50 & 3.09 & Sugars/Amino acids \\
4 & 3.94 & 3.09 & Sugars/Amino acids \\
5 & 3.42 & 2.79 & Sugars/Amino acids \\
\hline${ }^{1}$ VIP = Variable importance plot. & &
\end{tabular}

shown in Figure 4.

Anaerobic fungi possess both reductive and oxidative branches of the TCA cycle in the cytosol, producing succinate and $\alpha$-ketoglutarate respectively as proposed by Kwon et al. (2009). In the present study, succinate was

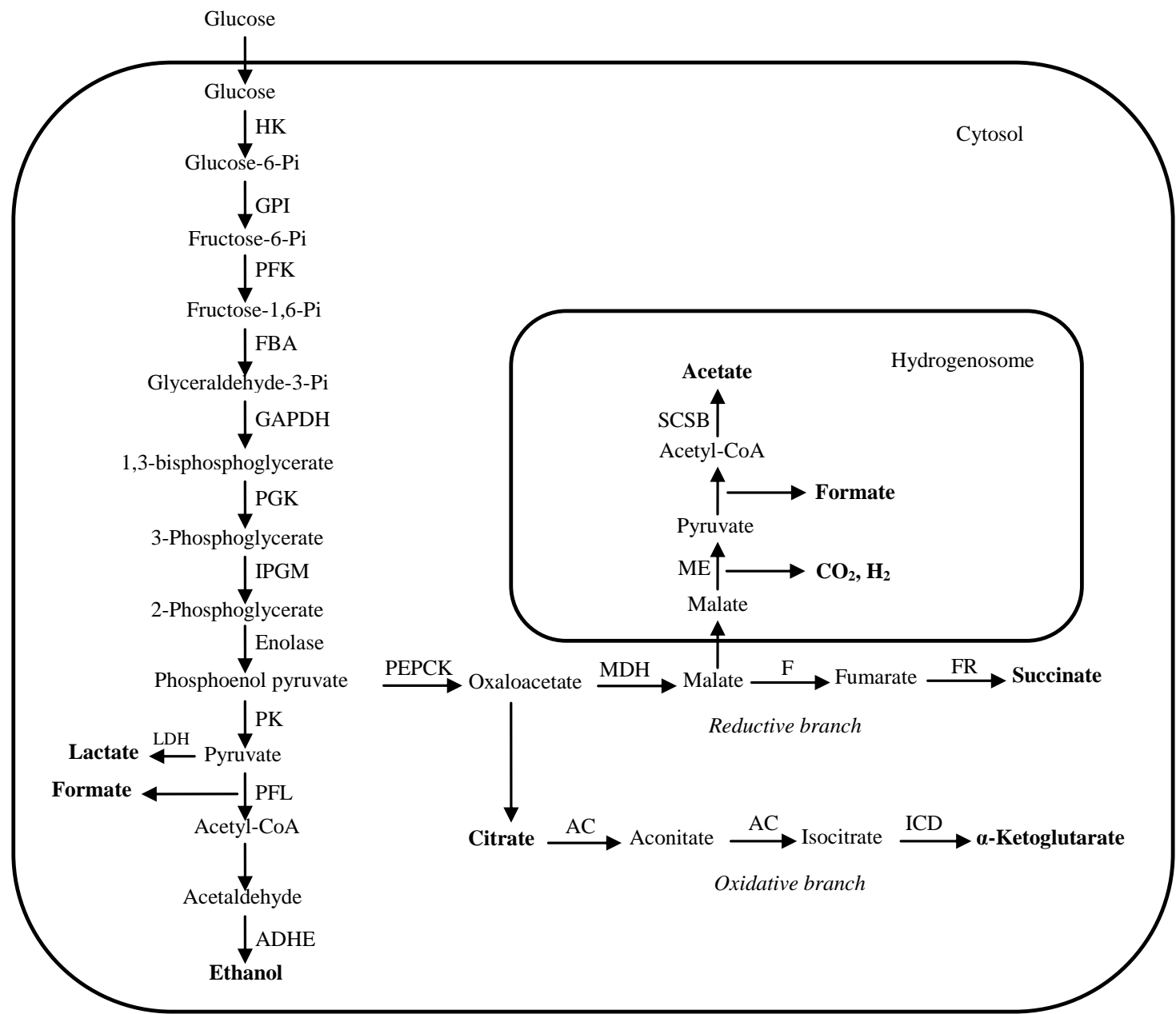

Figure 4. Metabolic pathway of carbohydrate by anaerobic fungi. Metabolic pathway of carbohydrate by anaerobic fungi based on previous reports by O'Fallon et al. (1991), Akhmanova et al. (1999), Boxma et al. (2004) and Kwon et al. (2009). AC = Aconitase; ADHE = Aldehyde/alcohol dehydrogenase $; \mathrm{F}=$ Fumarase $; \mathrm{FBA}=$ Fructose-bisphosphate aldolase $; \mathrm{FR}=$ Fumarate reductase $; \mathrm{GAPDH}=$ Glyceraldehydes 3-phosphate dehydrogenase; GPI = Glucose-6-phosphate isomerase; HK = Hexokinase; ICD = Isocitrate dehydrogenase; IPGM = 2,3-bisphosphoglycerate-independent phosphoglycerate mutase; $\mathrm{LDH}=$ Lactate dehydrogenase; $\mathrm{MDH}=\mathrm{Malate}$ dehydrogenase; $\mathrm{ME}=$ Malic enzyme; PEPCK = Phosphoenolpyruvate carboxykinase; PFK = Phosphofructokinase PFL = Pyruvate formate lyase; PGK = 3-phosphoglycerate kinase; PK = Pyruvate kinase; SCSB = Succinyl-CoA synthetase, beta subunit. 
detected in lower amounts than $\alpha$-ketoglutarate in anaerobic fungal monoculture, suggesting that the reductive branch was less active than the oxidative branch of the TCA cycle in the cytosol of fungal monoculture. However, succinate production increased and $\alpha$-ketoglutarate decreased dramatically in the co-cultures, implying that the reductive branch was stimulated, and the oxidative branch was inhibited, resulting in the accumulation of citrate. The citrate synthase, converting oxaloacetate to citrate, has not been detected until now, even though Kwon et al. (2009) postulated that there might be a possible existence of citrate synthase. The co-culture system in this study might be a useful tool for investigating the citrate formation in anaerobic fungi. Meanwhile, the oxidation reactions in the cytosol of anaerobic fungi and effects of co-cultured methanogens on these reactions need much more investigation.

In the hydrogenosome of anaerobic fungi, the metabolic pathway was also stimulated as revealed by increased acetate production. Results in this study showed that the acetate production in the co-cultures was twice that in the monoculture. According to previous reports, the $\mathrm{H}_{2}$ formed in the hydrogenosome was provided for the growth of cocultured methanogens, and cannot be detected in the cocultures (Marvin-Sikkema et al., 1990; Teunissen et al., 1992). Until now, the knowledge of the metabolic pathway in the hydrogenosome of anaerobic fungi was limited. And thus it is difficult to analyze the effects of associated methanogens on the metabolic pathway in the hydrogenosome of the fungi. In the future, the metabolism in the hydrogenosome of anaerobic fungi needs to be further investigated in mono- and co-cultures.

This study was the first to investigate the metabolism of anaerobic fungi by metabolomics and the novel finding of citrate as one of the major metabolites of anaerobic fungi co-cultured with methanogens by NMR may suggest a new yet unknown pathway in the co-culture. However, the metabolic pathway of anaerobic fungi, especially in the presence of methanogens, needs to be further investigated in the cytosol as well as in the hydrogenosome. The present study also showed that the anaerobic fungi were important electron contributors for methanogens in the rumen and their contribution to ruminal methane emission needs to be assessed.

\section{ACKNOWLEDGEMENTS}

This work was supported financially by the Natural Science Foundation of China (Grant No. 31101735, 31072052), China-Australia Project (2010DFA31040) and the Fundamental Research Funds for the Central Universities (KYZ201312).

\section{REFERENCES}

Akhmanova, A., F. G. J. Voncken, K. M. Hosea, H. Harhangi, J. T. Keltjens, H. J. M. op den Camp, G. D. Vogels, and J. H. P. Hackstein. 1999. A hydrogenosome with pyruvate formatelyase: anaerobic chytrid fungi use an alternative route for pyruvate catabolism. Mol. Microbiol. 32:1103-1114.

Balch, W. E., G. E. Fox, L. J. Magrum, C. R. Woese, and R. S. Wolfe. 1979. Methanogens: reevaluation of a unique biological group. Microbiol. Rev. 43:260-296.

Bauchop, T. and D. O. Mountfort. 1981. Cellulose fermentation by a rumen anaerobic fungus in both the absence and the presence of rumen methanogens. Appl. Environ. Microbiol. 42:11031110.

Beckonert, O., H. C. Keun, T. M. Ebbels, J. Bundy, E. Holmes, J. C. Lindon, and J. K. Nicholson. 2007. Metabolic profiling, metabolomic and metabonomic procedures for NMR spectroscopy of urine, plasma, serum and tissue extracts. Nat. Protoc. 2:2692-2703.

Boxma, B., F. Voncken, S. Jannink, T. van Alen, A. Akhmanova, S. W. van Weelden, J. J. van Hellemond, G. Ricard, M. Huynen, A. G. Tielens, and J. H. Hackstein. 2004. The anaerobic chytridiomycete fungus Piromyces sp. E2 produces ethanol via pyruvate: formate lyase and an alcohol dehydrogenase E. Mol. Microbiol. 51:1389-1399.

Carrieri, D., K. McNeely, A. C. De Roo, N. Nennette, I. Pelczer, and G. C. Dismukes. 2009. Identification and quantification of water-soluble metabolites by cryoprobe-assisted nuclear magnetic resonance spectroscopy applied to microbial fermentation. Magn. Reson. Chem. 47:S138-S146.

Cheng, Y. F., J. E. Edwards, G. G. Allison, W. Y. Zhu, and M. K. Theodorou. 2009. Diversity and activity of enriched ruminal cultures of anaerobic fungi and methanogens grown together on lignocellulose in consecutive batch culture. Bioresour. Technol. 100:4821-4828.

Cheng, Y. F., S. Y. Mao, C. X. Pei, J. X. Liu, and W. Y. Zhu. 2006. Detection and diversity analysis of rumen methanogens in cocultures with anaerobic fungi. Acta Microbiologica Sinica 46: 879-883.

Chikayama, E., M. Suto, T. Nishihara, K. Shinozaki, T. Hirayama, and J. Kikuchi. 2008. Systematic NMR analysis of stable isotope labeled metabolite mixtures in plant and animal systems: coarse grained views of metabolic pathways. PLoS ONE 3: e3805.

Grivet, J. P. 2001. NMR and microorganisms. Curr. Issues Mol. Biol. 3:7-14.

Grivet, J. P. and A. M. Delort. 2009. NMR for microbiology: In vivo and in situ applications. Prog. Nucl. Magn. Reson. Spectrosc. 54:1-53.

Hungate, R. E. 1982. Methane formation and cellulose digestion biochemical ecology and microbiology of the rumen ecosystem. Experimenta 38:189-192.

Jin, W., Y. F. Cheng, S. Y. Mao, and W. Y. Zhu. 2011. Isolation of natural cultures of anaerobic fungi and indigenously associated methanogens from herbivores and their bioconversion of lignocellulosic materials to methane. Bioresour. Technol. 102:7925-7931.

Joblin, K. N., G. E. Naylor, and A. G. Williams. 1990. Effect of Methanobrevibacter smithii on xylanolytic activity of 
anaerobic ruminal fungi. Appl. Environ. Microbiol. 56:22872295.

Kwon, M., J. Song, J. K. Ha, H. S. Park, and J. Chang. 2009 Analysis of functional genes in carbohydrate metabolic pathway of anaerobic rumen fungus Neocallimastix frontalis PMA02. Asian-Aust. J. Anim. Sci. 22:1555-1565.

Liu J. H. 2009. Metabolic profiles of natural co-cultures of anaerobic fungi and methanogens in vitro and the production of lactate. Ma.D. Thesis, Nanjing Agricultural University, Nanjing, China.

Marvin-Sikkema, F. D., A. J. Richardson, C. S. Steward, J. C. Gottschal, and R. A. Prins. 1990. Influence of hydrogenconsuming bacteria on cellulose degradation by anaerobic fungi. Appl. Environ. Microbiol. 56:3793-3797.

Mountfort, D. O., R. A. Asher, and T. Bauchop. 1982. Fermentation of cellulose to methane and carbon dioxide by a rumen anaerobic fungus in a triculture with Methanobrevibacter sp. Strain RA1 and Methanosarcina barkeri. Appl. Environ. Microbiol. 44:128-134.

Nakashimada, Y., K. Srinivasan, M. Murakami, and N. Nishio. 2000. Direct conversion of cellulose to methane by anaerobic fungus Neocallimastix frontalis and defined methanogens. Biotechnol. Lett. 22:223-227.
Orpin, C. G. 1975. Studies on the rumen flagellate Neocallimastix frontalis. J. Gen. Microbiol. 91:249-262.

O'Fallon, J. V., R. W. Wright, and R. E. Calza. 1991. Glucose metabolic pathways in the anaerobic rumen fungus Neocallimastix frontalis EB188. J. Biochem. 274:595-599.

Pham, L. H., J. Vater, W. Rotard, and C. Mugge. 2005. Identification of secondary metabolites from Streptomyces violaceoruber TU22 by means of on-flow LC-NMR and LCDAD-MS. Magn. Reson. Chem. 43:710-723.

Teunissen, M. J., E. P. W. Kets, H. J. M. Op den Camp, J. H. J. Huis in't Veld, and G. D. Vogels. 1992. Effect of coculture of anaerobic fungi isolated from ruminants and non-ruminants with methanogenic bacteria on cellulolytic and xylanolytic enzyme activities. Arch. Microbiol. 157:176-182.

Theodorou, M. K., D. R. Davies, and C. G. Orpin. 1995. Nutrition and survival of anaerobic fungi. In: Anaerobic fungi: Biology, Ecology and Function (Ed. D. O. Mountfort and C. G. Oprin). Marcel Dekker, New York, USA. pp. 107-128.

Theodorou, M. K., G. Mennim, D. R. Davies, W. Y. Zhu, A. P. Trinci, and J. L. Brookman. 1996. Anaerobic fungi in the digestive tract of mammalian herbivores and their potential for exploitation. Proc. Nutr. Soc. 55:913-926. 